\title{
La Universidad Internacional en América Latina: un Nuevo Paradigma para el Siglo XXI
}

\author{
Martha A. S. Lucchesi \\ Universidad de São Paulo (USP), Núcleo de Pesquisa de Políticas Públicas, \\ Rua do Anfiteatro, 181, f. 9.CEP:05508-060 São Paulo, SP, Brasil (mgrlucchesi@uol.com.br )
}

Recibido Dic. 30, 2010; Aceptado Feb. 07, 2011; Versión final recibida Feb. 15, 2011

\begin{abstract}
Resumen
Se discute y analiza el tema de la internacionalización de la universidad brasileña en el ámbito de América Latina. El objetivo es estudiar las contribuciones de esta internacionalización a la universidad, cuya episteme y pertinencia es la producción y el intercambio del conocimiento científico, considerando que el conocimiento se convirtió en un bien permanente en la posmodernidad. Las técnicas empleadas fueron la investigación bibliográfica, el análisis documental, y para la investigación cuantitativa, el método de encuestas. La universidad siempre fue supranacional, teniendo como misión la de continuar siendo la productora y socializadora del conocimiento, razón de ser de su historia, definición de su episteme y justificativa de su ethos.
\end{abstract}

Palabras clave: universidad, internacionalización, producción del conocimiento, paradigma, posmodernidad

\section{The International University in Latin America: a New Paradigm for XXI Century}

\begin{abstract}
The subject of internationalization of the Brazilian university in Latin America is discussed and analyzed. The goal is to study the contributions of this internationalization for the university, which relevance and episteme is the production and exchange of scientific knowledge, considering that knowledge has become a permanent value in the post-modern world. The techniques employed were literature search, document analysis and quantitative research by survey. The university has always been supra-national, being its mission to continue being the source of knowledge production and diffusion, reason of its history, definition of its episteme and justification of its ethos.
\end{abstract}

Keywords: university, internationalization, knowledge production, paradigm, post-modernity 


\section{INTRODUCCIÓN}

Con el advenimiento de la globalización se anuncia una nueva era, la del conocimiento o sociedad de los saberes. La etimología de "saber" está vinculada a la raíz indoeuropea "sap", "saber" y "tener el sabor de", de donde se originan palabras como "sabiduría", "sapiencia". El saber remite a la teoría a partir de diferentes concepciones filosóficas; diferente de la noción de sociedad de la información guiada apenas por la tecnología, las "sociedades de conocimiento" son "minddriven", guiadas por el espíritu. Por otra parte, la recusa en recurrir a la noción singular de "sociedad global" y la adopción de la noción plural de "sociedades" ratifica la idea de que los modos de apropiación de las tecnologías son resultado de la diversidad de las configuraciones de actores inscriptos en los contextos institucionales, culturales, industriales y políticos (Mattelart, 2005). En síntesis, es reconocida la especificidad de los "regímenes epistémicos, y la educación pasa a ser considerada el centro de la agenda disponible para que los Estados (nacionales 0 transnacionales) enfrenten esa nueva estructuración del mundo. La Educación Superior representa la base de la continuidad del proceso de desarrollo económico y justicia social y frente a este contexto trata de internacionalizarse. Así, en la era planetaria, los formuladores de las políticas públicas (policy formulation) de la educación brasileña inician proyectos para colocar en práctica el indispensable proceso de intercambio del conocimiento entre los países.

Castells (1999) afirma que el mundo que surgió a partir del final del siglo XX se fundamentó en una coincidencia de la aceleración de la información con algunas crisis esenciales del sistema. Él afirmaba, ya al final del siglo pasado, que un mundo nuevo se estaba configurando en ese final de milenio. Las transformaciones comenzaron poco después de la mitad del siglo XX, en las décadas de 1960 y 1970, cuando ocurrieron, simultáneamente, aunque de forma independiente: la "revolución de la tecnología de la información", la "crisis económica del capitalismo y del estatismo" y el "apogeo de los movimientos sociales y culturales" (Castells, 1999). Afirma también que la "revolución de la tecnología de la información motivó el surgimiento del informacionalismo como la base material de una nueva sociedad". El chama de "sociedad red" el resultado de la interacción entre los procesos descriptos más arriba, que produjeron una nueva economía que caracteriza como informacional (basada en la informática y en la red internacional de computadores WEB) y por consecuencia, global. La sociedad red también impuso una nueva cultura, "la cultura de la virtualidad real". Siguiendo con Castells, la "lógica insertada en esa economía, en esa sociedad y en esa cultura está subyacente a la acción y a las instituciones sociales en un mundo interdependiente."

Para Carnoy (2006) las dos bases de la globalización son la información y la innovación, basadas en el conocimiento. Sin embargo, hay que distinguir qué tipo de conocimiento puede ser necesario en el ambiente "líquido moderno" enunciado por Bauman (2005) para que éste pueda influenciar las fuerzas sociales y difundir, más allá del conocimiento técnico científico, condiciones de ciudadanía. El contexto denominado por Bauman como posmoderno, considerando la velocidad de los cambios y la era de las incertidumbres, obliga a la universidad a repensar sus funciones y a buscar en su interior, en la comunidad académica, una nueva episteme que deberá provocar una ruptura y una transición paradigmática.

Los fenómenos de ámbito mundial también se dan en la región de América Latina y el Caribe. Esto ocurre en una doble dirección, como históricamente ha ocurrido con los países de cultura ibérica de América del Sur y Central y con los países de diferentes influencias culturales en el Caribe: la relación internacional se da primeramente "vía Océano Atlántico" y solamente después con los vecinos. Brasil ha buscado el apoyo europeo para el desarrollo de sus modelos internacionales de educación. Aunque este hecho constituye un avance, existe la convicción de que la mayor implementación de este desarrollo se dará ahora con la integración interamericana. Sin embargo no se ha alcanzado el mismo estándar europeo por la falta de tradición de la enseñanza superior, que remonta a aproximadamente cien años, en comparación con los casi mil años de las más antiguas universidades medievales en Europa. Como dice Morin (2007), la universidad europea moderna es una "dádiva de la Edad Media". Aunque con atraso en relación al 
proceso europeo, Brasil realiza actualmente el comienzo de la internacionalización a través del intercambio de estudiantes e investigadores.

En la Conferencia Regional de Educación Superior en América Latina y el Caribe (CRES), realizada del 4 al 6 de junio de 2008 en la ciudad de Cartagena de Indias, Colombia, se evidenció que la misión de expandir la enseñanza superior en los países de América Latina y el Caribe es un gran desafío, ya sea por la urgencia, ya sea por la amplitud de todo lo que es necesario crear e implementar. Esa tarea incluye "tanto el sector público como el privado", que "están obligados a otorgar una Educación Superior con calidad y pertinencia". De acuerdo con Sampaio (2000) "la relación de complementariedad existente en Brasil entre el sistema público y el sistema privado de enseñanza superior se consolidó en el período de mayor expansión de este nivel de enseñanza."A pesar de todo esto, hay poco intercambio o diálogo entre las diferentes categorías administrativas, inclusive cuando son públicas. Este punto nos interesa particularmente en esta investigación porque creemos firmemente que habiendo estudiado y defendido en los últimos años la propuesta de un nuevo paradigma para la universidad del tercer milenio, la excelencia y la pertinencia de la enseñanza superior están en el núcleo de nuestro objeto de estudio. Por lo tanto, el objetivo de este trabajo es estudiar las contribuciones de la internacionalización de la universidad en América Latina y el Caribe, resaltando la Universidad del Siglo XXI, cuya episteme y pertinencia es la producción e intercambio del conocimiento científico, considerando que el conocimiento se tornó un bien permanente para el "sujeto" de la posmodernidad.

En este trabajo se delimita en primer lugar el contexto mundial dialogando con Castells (1999), Bauman $(1999,2005)$ y otros autores que demuestran que un nuevo orden educacional debe insertarse dentro del nuevo orden mundia. En segundo lugar, se estudia las políticas públicas y el proceso de internacionalización propuesto para la educación superior brasileña, sobre la base del análisis documental de la Declaración de la Conferencia Regional de Educación Superior en América Latina y el Caribe, realizada en Cartagena de Indias, Colombia, el año 2008. Después, se discuten las propuestas de integración para las universidades latinoamericanas y del Caribe y sus consecuencias; y se analiza los datos sobre los números de Instituciones de Educación Superior (IES) en los países del Cono Sur. Se muestran datos de IES y de estudiantes de Brasil, al mismo tiempo que se presenta también el ethos de la universidad: la investigación como el paradigma posible para la Universidad del comienzo del siglo XXI. El texto finaliza con algunas consideraciones sobre el tema investigado.

\section{REFLEXIONES SOBRE LA UNIVERSIDAD}

El conocimiento creado por las instituciones de enseñanza superior debe incluir la reflexión sobre la sociedad como respuesta a las demandas de esta sociedad en la que se insertan, y también la rigorosa autocrítica, para que la comunidad universitaria sepa definir sus finalidades y asumir sus compromisos. Para que la universidad cumpla su función es fundamental la libertad académica que incluye el poder de decisión sobre sus prioridades, manteniendo, naturalmente, la legalidad y los principios que fundamentan la ciencia y el bienestar social. En la Conferencia Regional de Educación Superior en América Latina y el Caribe (CRES) de 2008 se resaltó que "los gobiernos deben fortalecer los mecanismos de reconocimiento que garanticen la transparencia y la condición de servicio público", teniendo en vista la especial relevancia de la educación superior en la producción de conocimiento científico, factor esencial de desarrollo y riqueza en el mundo globalizado. Paralelamente, es necesario que la educación superior organizada internacionalmente en la región contribuya para "la convivencia democrática, tolerancia y promoción de un espíritu de solidaridad y de cooperación" entre las naciones que la comparten.

Uno de los aspectos destacados por el documento de la conferencia "CRES" fue la importancia de la creación del conocimiento para la superación de la injusticia social, "para la transformación social y productiva de nuestras sociedades"...."En un continente que ostenta la penosa circunstancia de tener las mayores desigualdades sociales del planeta, los recursos humanos y el conocimiento serán las principales riquezas de todas las que dispongamos." (UNESCO, 2008). 
Cuando la universidad investiga produce información e innovación. La información que produce es de excelencia, es conocimiento científico y colabora para la producción de la riqueza nacional y mundial. La Universidad puede realizar también otras transformaciones: Se espera que al desempeñar su responsabilidad social, ella sea el gran instrumento de cohesión social (Lucchesi, 2002) beneficiando a la sociedad. Para eso, la Universidad debe buscar ahora la integración internacional con países vecinos y con identidades culturales semejantes. Si la economía, como dice Bauman (1999), se tornó supranacional, es natural que surjan nuevas formas de organización geopolítica que reúnan varios países. Afirma Ferrer (2009) "Nadie puede profundizar su desarrollo sin mejorar su productividad y su cohesión social, pero tampoco puede hacerlo en situación de aislamiento. Aunque se ha hablado mucho de la necesidad de establecer mecanismos regionales de cooperación, las circunstancias actuales lo hacen aún más necesario."

La internacionalización de la universidad brasileña se ha realizado a través de dos modelos: el de las instituciones privadas, mediante acuerdos, fusiones y adquisiciones y el de los proyectos del Ministerio de Educación - MEC con el intercambio de estudiantes e investigadores entre los países de América Latina y otros en desarrollo. Existe también el objetivo de integrar la educación superior a través de una universidad federal (UNILA) ya fundada en 2010 en la triple frontera entre Brasil, Paraguay y Argentina. Dentro de este contexto y teniendo en cuenta su importancia en la esfera de las políticas públicas y sus consecuencias para la cultura nacional, algunos países en desarrollo como Brasil tratan de implementar una agenda política en la que el intercambio del conocimiento entre investigadores y estudiantes contribuya para la integración y el desarrollo de los países involucrados.

Finalmente, cabe a la nueva universidad de América Latina y el Caribe la construcción de una identidad continental que tenga en cuenta las similitudes entre todos los países que componen este bloque internacional, sin anular las peculiaridades de cada pueblo y nación. De acuerdo con Ferrer (2009) "La construcción del Espacio Iberoamericano del Conocimiento (EIC) es una condición necesaria para promover el desarrollo iberoamericano, considerando su contribución al incremento de la productividad y de la competitividad de los países de la región." Es fundamental también que este movimiento de crecimiento de la educación superior en América Latina y el Caribe genere oportunidades para los que no tienen acceso a la enseñanza superior y contribuya para una mayor justicia social en toda la región.

Carnoy (2006) afirma que: "El aumento de la demanda por la educación superior es el resultado de la exigencia de niveles más elevados de educación y de la mejor remuneración obtenida por los que tienen formación superior." Si desde la antigüedad hasta la mitad del siglo XX la enseñanza superior era destinada solamente a una elite social, económica e intelectual, las necesidades contemporáneas exigen que una parte mayor de la población económicamente activa tenga acceso a éste grado de enseñanza así como a los posteriores. Al mismo tiempo es esencial la capacidad de producir conocimiento nuevo que tenga, dentro de lo posible, difusión y aceptación internacional. La contribución de este estudio para el desarrollo de la universidad internacional en América Latina consiste en proponer un nuevo paradigma, la "universidad emergente" (Lucchesi, 1999). Según Morin (2001), un paradigma es aquello que delinea y define un cuadro epistémico. Es un marco conceptual, un conjunto de referencias capaz de conducir las investigaciones científicas durante un cierto período. Puede ser un constructo que ponga fin a controversias existentes en una determinada área en relación a ciertos fundamentos o puede ser el "propio principio de organización de las teorías".

Para Castells (1999) "la globalización no significó solamente mayor rapidez de la circulación internacional del capital financiero, de la producción, de las personas y de los servicios; también generó una aceleración de la producción, difusión e intercambio del conocimiento", porque si el intercambio internacional del conocimiento científico ya ocurría desde la Antigüedad y durante la Edad Media, éste se intensificó a partir de la modernidad. La globalización posmoderna no modificó este modelo pero lo aceleró y lo intensificó a tal punto que "generó cambios en el proceso de producción del conocimiento científico", principalmente "debido a la posibilidad de superar distancias", con la formación de networks mundiales de investigadores". (Lucchesi, 2009) 
Los análisis muestran que producir y difundir conocimiento es la función social de la universidad. La aplicación de ese conocimiento en beneficio de la colectividad es parte de la misión de la universidad. Por ejemplo, cuando realiza investigaciones sobre las relaciones entre el hombre, el medio natural y la economía en las regiones de preservación ambiental, la universidad está sirviendo al mismo tiempo a la comunidad local y a toda la humanidad, cumpliendo así su finalidad de universalidad, pues es universitas. Cuando los aspectos económicos de la globalización se encuentran en crisis, la circulación del conocimiento permanece como el gran eslabón de la globalización del inicio del siglo XXI. Y para el mundo académico es fundamental que sea el conocimiento científico el que esté al frente de este tiempo conceptualizado por Bauman como "posmodernidad". Para este autor, ésta "es la condición actual de la modernidad"(Bauman, 1999). La producción y la difusión del conocimiento son funciones sociales esenciales de la universidad. Ella se define por estas funciones. Por otro lado, aplicar el conocimiento producido en beneficio de la sociedad es una misión de la universidad.

\section{RESULTADOS Y DISCUSIÓN}

Aunque en general la educación superior en América Latina presenta gran diversidad, el desafío de las políticas públicas es integrarla. Esta diversidad existe inclusive dentro del ámbito del MERCOSUR, donde la educación superior presenta gran "heterogeneidad" (Lucchesi, 2007). Mencionando una vez más la Declaración de la Conferencia Regional de Educación Superior en América Latina y el Caribe (2008), tanto el sistema público como el privado de educación superior deben participar de la tarea de la integración internacional de la universidad latinoamericana y caribeña. Pero "el término privatización ha sido usado para designar experiencias muy distintas: desde el aumento de la participación relativa de las matrículas privadas, sobre todo en los países con una larga tradición en la atención pública de la demanda de enseñanza superior (Argentina y México)" hasta la "cobranza de tasas de matrículas y de anualidades en las universidades públicas (Chile a principios de los años 80 y más recientemente Colombia)", o inclusive "la formulación de las políticas para la enseñanza superior en esos países".(Durham y Sampaio, 2000).

A partir de la década de 1970 se observa en Brasil una significativa ampliación de la participación del sector privado, lo que no ocurre en los demás países de América Latina. "Entre 1960 y 1980 hubo una gran expansión de la enseñanza superior en América Latina, pero de forma desigual. A pesar de la expansión de la tasa de matrículas, Brasil continuó con un contingente menor de estudiantes. En 1986, Argentina, Ecuador, Costa Rica y Venezuela llegaron a tener más del 20\% de los estudiantes en el curso superior." (Lucchesi, 2007). El mayor contingente de estos estudiantes estaba en instituciones públicas.

Es interesante observar la figura 1, que hace una comparación de los tipos de instituciones de enseñanza superior que existen en algunos de los principales países de América del Sur (Brasil, Argentina, Chile, Uruguay y Paraguay). Debemos destacar que en Brasil el Censo de la Educación Superior de 2007 (BRASIL, 2009) registró la participación de 2.281 IES. La distribución de IES por categorías administrativas se aproxima de la verificada en el año anterior, con 89\% de instituciones privadas y $11 \%$ de instituciones públicas, divididas entre federales (4,6\%), estaduales $(3,6 \%)$ y municipales $(2,7 \%)$, incluyendo todas las IES que ofrecen cursos de graduación (presencial y a distancia). Los datos son provenientes de los ministerios de Educación de los países citados y fueran colectados por la autora en investigación realizada en 2009.

Aunque hubo un incremento de $2,8 \%$ en el número de universidades en relación a 2006, ellas aún representan solamente el 5,3\% del total de instituciones superiores de enseñanza en el país. Los centros universitarios corresponden al $8 \%$ del total, mientras que las facultades (facultades, escuelas, institutos, facultades integradas, centros federales de educación tecnológica y facultades de tecnología - Decreto n. 5.773/2006) mantuvieron la superioridad como en años anteriores, con cerca de 2.000 establecimientos, correspondientes al $86,7 \%$ de las IES (BRASIL, 2009). 


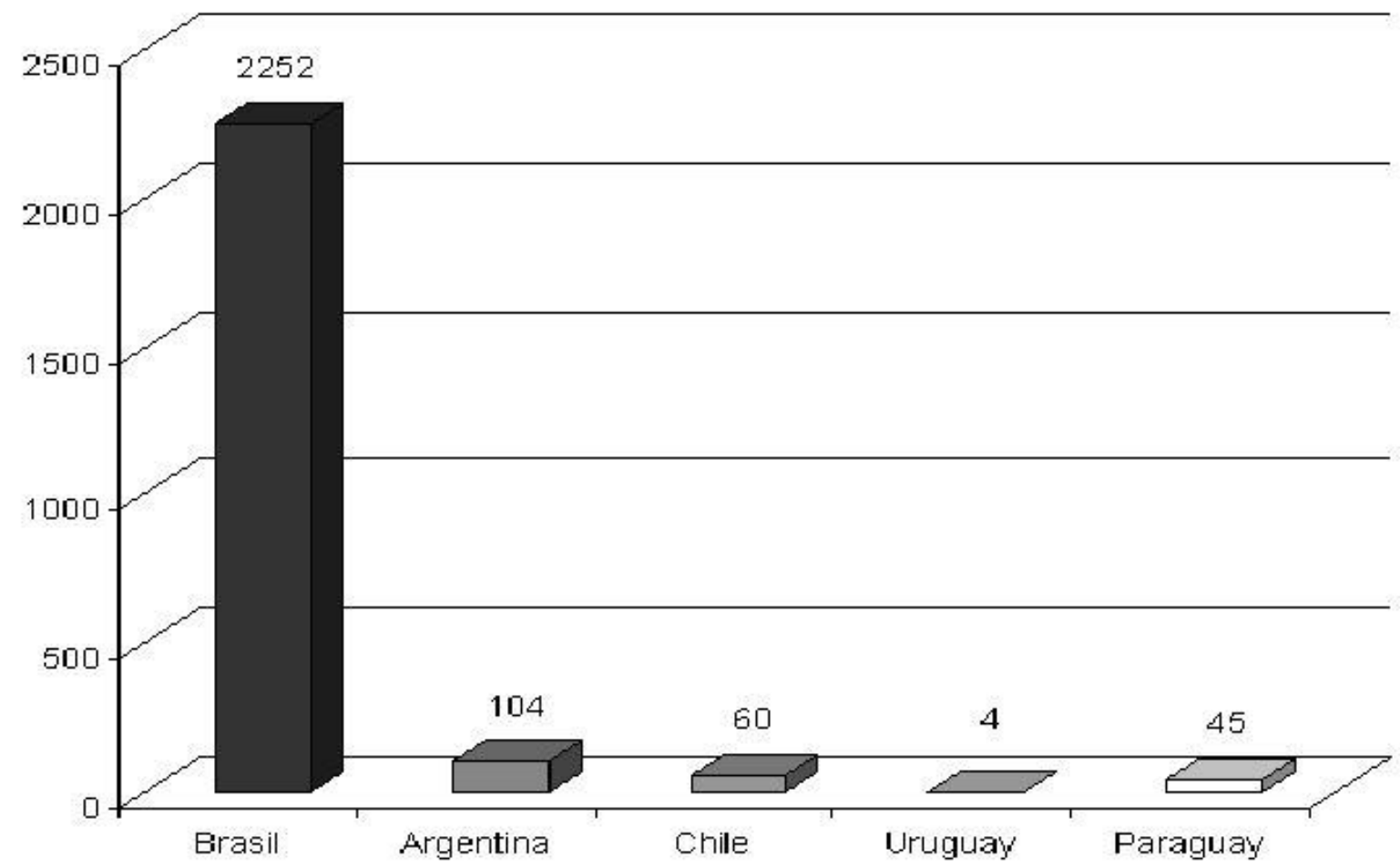

Fig. 1: Comparación de los tipos de instituciones de enseñanza superior que existen en algunos de los principales países de América del Sur

En Chile todas las universidades son fundaciones de derecho público (estatales). En este país se canceló la gratuidad en la educación pública, lo que posibilitó la expansión del sistema privado. Cuenta también con 44 Institutos Profesionales y 72 Centros de Formación Técnica. En Argentina, sobre 1.567.519 estudiantes en 2007 (contando también a los nuevos inscriptos y egresados), el 81,06 \% (1.270.755) cursa sus estudios en el sector estatal y el restante 18,93\% (296.764) en el privado. Pero al mismo tiempo la tasa promedio de crecimiento anual fue del 7,2 \% para el sector privado mientras que para el público fue solo de un $3,8 \%$. Debemos agregar que en diez años, el crecimiento de la población estudiantil fue solo un 4,4 \%. En 2007 hubo 87.102 egresados - contra 84.785 del 2006 -. De ese número total, 63.046 egresaron de universidades públicas y 24.056 de privadas (es importante señalar que estos números fueron solo un poco más elevados que los de 2006). La tasa promedio de crecimiento anual de los egresados - entre 1997 y 2007 - fue del 6,5 \%. En el Uruguay la cantidad de alumnos en universidades e institutos universitarios es de 97.199 .

Los datos de la Tabla 1 revelan que el predominio del sector privado es mayor en São Paulo que en el resto de Brasil, debido a dos factores:

a) En el Estado se concentra una población con ingresos más elevados. Por lo tanto, hay una demanda más grande de enseñanza particular.

b) La participación del gobierno federal en el financiamiento de instituciones públicas de enseñanza superior en el Estado de São Paulo es relativamente baja - al contrario de lo que se observa en las demás unidades de la Federación. Las tres universidades públicas son financiadas con recursos estaduales. 
Tabla 1: Comparación del número de alumnos en las Instituciones de Enseñanza Superior federales, estaduales, municipales y particulares en São Paulo y en Brasil en 2007. 1 Excluyendo matrículas en Centros de Educación Tecnológica (CETs) y Facultades de Tecnología (FaTs). Los datos son tomados de Microdados do Censo do Ensino Superior (INEP)

\begin{tabular}{|c|c|c|c|c|c|c|c|c|c|c|c|c|}
\hline \multirow{2}{*}{ Ano } & \multicolumn{2}{|l|}{ Federal } & \multicolumn{2}{|c|}{ Estadual } & \multicolumn{2}{|c|}{ Municipal } & \multicolumn{2}{|c|}{ Público } & \multicolumn{2}{|c|}{ Particular } & \multicolumn{2}{|l|}{ Total } \\
\hline & $\mathrm{N}^{\circ}$ & $\%$ & $\mathrm{~N}^{\circ}$ & $\%$ & $\mathrm{~N}^{\circ}$ & $\%$ & $\mathrm{~N}^{\circ}$ & $\%$ & $\mathrm{~N}^{\circ}$ & $\%$ & $\mathrm{~N}^{\circ}$ & $\%$ \\
\hline \multicolumn{13}{|c|}{ São Paulo } \\
\hline 1999 & 6,754 & 0.9 & 70,908 & 9.7 & 35,162 & 4.8 & 112,824 & 15.4 & \multicolumn{2}{|c|}{$618,69884.6$} & 731,522 & 100 \\
\hline 2000 & 7,114 & 0.9 & 70,595 & 8.7 & 37,875 & 4.7 & 115,584 & 14.3 & \multicolumn{2}{|c|}{$692,75185.7$} & 808,335 & 100 \\
\hline 2001 & 7,358 & 0.8 & 72,624 & 8.2 & 39,930 & 4.5 & 119,912 & 13.5 & \multicolumn{2}{|c|}{$767,53686.5$} & 887,448 & 100 \\
\hline 2002 & 7,570 & 0.8 & 81,176 & 8.3 & 53,301 & 5.5 & 142,047 & 14.6 & \multicolumn{2}{|c|}{$830,84685.4$} & 972,893 & 100 \\
\hline 2003 & 7,832 & 0.8 & 90,818 & 8.8 & 57,653 & 5.6 & 156,303 & 15.1 & \multicolumn{2}{|c|}{$876,12884.9$} & $1,032,431$ & 100 \\
\hline 2004 & 7,729 & 0.7 & 90,818 & 8.4 & 60,984 & 5.7 & 159,531 & 14.8 & \multicolumn{2}{|c|}{$919,79085.2$} & $1,079,321$ & 100 \\
\hline 2005 & 7,687 & 0.7 & 96,276 & 8.4 & 60,956 & 5.3 & 164,919 & 14.3 & \multicolumn{2}{|c|}{$985,10285.7$} & 1,150,021 & 100 \\
\hline 2006 & 8,111 & 0.7 & 95,956 & 7.8 & $60,72 \varepsilon$ & 4.9 & 164,795 & 13.4 & \multicolumn{2}{|c|}{$1,065,82086.6$} & $1,230,615$ & 100 \\
\hline 2007 & 10,139 & 0.8 & 98,606 & 7.5 & 59,295 & 4.5 & 168,040 & 12.8 & \multicolumn{2}{|c|}{$1,139,82887.2$} & $1,307,868$ & 100 \\
\hline \multicolumn{13}{|c|}{ Tasa de crecimiento (\%) } \\
\hline 1999-2001 & \multicolumn{2}{|l|}{8.9} & \multicolumn{2}{|l|}{-8.6} & \multicolumn{2}{|c|}{13.6} & \multicolumn{2}{|c|}{6.3} & \multicolumn{2}{|c|}{24.1} & \multicolumn{2}{|l|}{19.9} \\
\hline 2001-2006 & \multicolumn{2}{|l|}{10.2} & \multicolumn{2}{|l|}{32.1} & \multicolumn{2}{|c|}{52.1} & \multicolumn{2}{|c|}{37.4} & \multicolumn{2}{|c|}{38.9} & \multicolumn{2}{|l|}{38.7} \\
\hline 2006-2007 & \multicolumn{2}{|l|}{25.0} & \multicolumn{2}{|l|}{2.8} & \multicolumn{2}{|c|}{-2.4} & \multicolumn{2}{|c|}{2.0} & \multicolumn{2}{|c|}{6.9} & 6.3 & \\
\hline
\end{tabular}

Brasil excluido São Paulo

$\begin{array}{llllllllll}1999 & 424,91526.2 & 222,88113.8 & 51,9183.2 & 699,714 & 43.2 & 919,22556.8 & 1,618,939 & 100 \\ 2000 & 462,28324.8 & 251,54013.5 & 34,2971.8 & 748,120 & 40.2 & 1,114,46859.8 & 1,862,588 & 100 \\ 2001 & 475,37422.5 & 273,78013.0 & 39,3201.9 & 788,47437.3 & 1,323,36062.7 & 2,111,834 & 100 \\ 2002 & 497,80220.2 & 323,54013.1 & 51,1512.1 & 872,49335.4 & 1,591,34864.6 & 2,463,841 & 100 \\ 2003 & 525,46818.8 & 340,89312.2 & 68,9102.5 & 935,27133.5 & 1,859,61366.5 & 2,794,884 & 100 \\ 2004 & 531,11417.7 & 366,51012.2 & 71,0992.4 & 968,72332.2 & 2,039,25767.8 & 3,007,980 & 100 \\ 2005 & 543,62716.9 & 365,16611.3 & 74,2972.3 & 983,09030.5 & 2,236,82669.5 & 3,219,916 & 100 \\ 2006 & 550,32816.4 & 369,23811.0 & 76,9992.3 & 996,56529.8 & 2,351,32970.2 & 3,347,894 & 100 \\ 2007 & 569,87216.4 & 366,70610.6 & 83,3172.4 & 1,019,89529.4 & 2,444,48070.6 & 3,464,375 & 100\end{array}$

Tasa de crecimiento (\%)

\begin{tabular}{rrrrrrr}
\hline $1999-2001$ & 9.1 & 22.8 & -24.3 & 12.7 & 44.0 & 29.6 \\
$2001-2006$ & 15.8 & 34.9 & 95.8 & 31.4 & 77.7 & 58.5 \\
$2006-2007$ & 3.6 & -0.7 & 8.2 & 2.3 & 4.0 & 3.5 \\
\hline
\end{tabular}


Como se puede observar, la superioridad numérica del sector privado en Brasil es muy alta en relación a los otros países. Sin embargo, la enseñanza superior de excelencia se concentra en las tres universidades estatales, que se ubican entre las mejores del mundo: Universidad de São Paulo (USP), Universidad Estadual Paulista (Unesp) y Universidad Estadual de Campinas (Unicamp). La USP está localizada principalmente en la ciudad de São Paulo, capital del Estado, habiendo sido creado recientemente un nuevo campus en la zona este de la capital (la Ciudad Universitaria, donde están localizadas la mayoría de las instituciones de enseñanza y de investigación de la USP, se encuentra en la zona oeste de la capital). La USP ya inició su proceso de interiorización a través de seis campi. La Unesp está distribuida en 23 ciudades del Estado de São Paulo y la Unicamp se concentra en Campinas, una gran ciudad del interior de São Paulo, contando también con otros dos campi en expansión en ciudades menores. En la última edición de Shanghai University (2008), que clasifica las 500 mejores universidades del mundo, la USP obtuvo la $121^{a}$ posición. El índice de The Times es formado por las 200 instituciones académicas de mayor relevancia mundial. Allí, la Universidad de São Paulo obtuvo, en 2008, el $196^{\circ}$ lugar. El 2007 Performance Ranking of Scientific Papers for World Universities, del Higher Education Evaluation \& Accreditation Council of Taiwan, que también clasifica las 500 mejores instituciones de enseñanza e investigación del mundo, otorga a la USP la $94^{a}$ posición. En la última edición de Shanghai University (2008), la Universidad Estadual de Campinas (Unicamp), obtuvo la $286^{\circ}$ posición y la Universidad Estadual Paulista (Unesp) también consta entre las 500 mejores.

Es claro que la tasa de acceso a la enseñanza superior en Brasil es aún insatisfactoria comparada con la de los países desarrollados e inclusive con la de otros países de América Latina. Es preocupante el hecho de que las tasas de crecimiento en el período considerado estén disminuyendo en Brasil y aún más en São Paulo, el más importante y desarrollado estado de la Federación. El conglomerado de la educación superior del MERCOSUR manifiesta aspectos notables de heterogeneidad en sus dimensiones y también en su calidad y recursos. Por esa misma razón, el acuerdo de integración puede generar sinergias en beneficio de los países con menor grado de desarrollo relativo. (Lucchesi, 2007). Por otro lado, las diferencias en los criterios de selección son un problema a ser superado en el plano del reconocimiento mutuo de estudios, de la formación de los profesionales y de la movilidad estudiantil. Sin embargo, la integración entre los países del MERCOSUR ya está ocurriendo. Algunos proyectos del MEC dan los primeros pasos para la integración de los países en desarrollo. Resaltemos que, "la cuestión en América del Sur, y principalmente en Brasil, es la división entre las universidades públicas y las privadas. Por cuestiones históricas e inclusive por cuestiones legales, la enseñanza superior brasileña se divide en pública y privada, no siendo habitual la cooperación entre las dos categorías." (Lucchesi, 2008).

Para facilitar el intercambio, las naciones de Argentina y Brasil están implantando la enseñanza del portugués y del español, respectivamente, en su enseñanza básica. El Congreso Nacional de Argentina aprobó el 17 de diciembre de 2008 la Ley $\mathrm{n}^{0}$ 26.468, que determina la oferta del portugués como lengua extranjera en todas las escuelas secundarias del país y en las áreas fronterizas con Brasil a partir del nivel primario. En contrapartida, en Brasil rige la Ley 11.161/05: Las escuelas tenían plazo hasta 2010 para incluir la enseñanza del español como disciplina optativa en las escuelas públicas y particulares de nivel medio. El idioma también puede ser incluido en los currículos de los cuatro últimos años de la enseñanza fundamental, a partir de la $5^{\mathrm{a}}$ serie ( $6^{\circ}$ año), aunque en este caso su ofrecimiento no es obligatorio.

Las declaraciones conjuntas y las cartas de intención continúan. Aún cuando solo se efectivicen a medio plazo - en una visión optimista de la cuestión -, demuestran la intención de que se produzcan convergencias entre las políticas públicas de los países del Mercosur. Dice la Declaración Conjunta de Buenos Aires: "Los Ministros de Educación del Mercosur y representantes de las centrales sindicales del Cono Sur, reunidos en Buenos Aires, firmaron en este miércoles, 9/9/2010, la declaración conjunta de directrices necesarias para alcanzar la meta de universalización de la enseñanza media en toda la región."(MEC/2010). La equivalencia de estudios ya era realizada por las propias escuelas, tomando como base el número de años cursados. Sin embargo faltaba una norma que obligase a todos los países miembros o asociados al Mercosur (Brasil, Argentina, Uruguay, Paraguay, Bolivia y Chile). De este modo, el alumno no 
se perjudicaría al mudarse dentro del bloque y esto posibilitaría mayor movilidad para las familias. La equivalencia fue reglamentada con base en una tabla prevista en el protocolo de integración educativa y reconocimiento de certificados, títulos y estudios de nivel fundamental y medio no técnico entre los países que componen el bloque, acuerdo celebrado en 2002.

A pesar de las diferencias, los acuerdos prosiguen. Por ejemplo, aunque surge con atraso en relación a los países desarrollados, tenemos la propuesta de universalización de la enseñanza media. "La universalización de la escolaridad secundaria o media es una meta que permite avanzar en la efectiva concreción del derecho a la educación en los países del MERCOSUR, y constituye una responsabilidad indelegable de los Estados y un desafío para la sociedad."(Buenos Aires, 2010). Las equivalencias de estudios y de títulos deben ser establecidas no sólo en la enseñanza básica (fundamental y media) sino también en el nivel superior, de modo que el extranjero que estudia en Brasil pueda volver a su país y ejercer la profesión para la cual se preparó. Por eso, ya hay grupos de trabajo estudiando la integración de la educación superior en el ámbito del Mercosur. El objetivo es crear un espacio académico regional. Para estimular el proceso de integración se considera necesario mejorar la calidad de la integración de los recursos humanos.

Debemos destacar que el tema de mayor controversia dentro de la agenda del MERCOSUR para la educación superior ha sido el del mutuo reconocimiento de títulos y grados dentro del espacio comunitario. Esto es causado principalmente por el hecho de que los países signatarios poseen sistemas educativos con estructuras diferentes, distintos criterios para la admisión y formación universitaria y volúmenes muy heterogéneos de matrículas universitarias y de número de profesionales, científicos y técnicos. También se espera obtener un sistema de mutuo reconocimiento de títulos y carreras para posibilitar una mayor movilidad internacional en la región e inclusive la cooperación interinstitucional, como consta de la Declaración de Cartagena (2008) se recomienda "el fortalecimiento del proceso de convergencia de los sistemas de evaluación y revalidación nacionales y subregionales".

El acuerdo Mercosur/cmc/dec. $N^{\circ} 17 / 08$ establecido entre los Estados partes es un avance en las negociaciones de reconocimiento y acreditación de las IES, a saber: "La acreditación en el sistema Arcu-Sur será impulsada por los estados partes del Mercosur y los estados asociados, como criterio común para facilitar el reconocimiento mutuo de títulos o diplomas de grado universitario para el ejercicio profesional, en convenios, tratados o acuerdos bilaterales, multilaterales, regionales o subregionales que vengan a ser celebrados a ese respecto." (Brasil, 2008). Recordemos que el objetivo de esta integración es la "garantía de calidad de la Educación Superior y de la investigación". O sea, las declaraciones conjuntas reconocen que no es posible pensar en educación superior de calidad sin tener como base la investigación que es la garantía de esa calidad de la enseñanza y del cumplimiento de "su función social y pública".

Los acuerdos y mecanismos de revalidación de diplomas y créditos académicos tienen como objetivo "el fomento de la movilidad interregional de estudiantes, investigadores, profesores y personal administrativo, inclusive mediante la implementación de fondos específicos" y está previsto "el emprendimiento de proyectos conjuntos de investigación y la creación de redes de investigación y docencia multiuniversitarias y pluridisciplinares". En El espacio iberoamericano del conocimiento: retos y propuestas, Ferrer propone el fomento al trabajo en redes: "El concepto de red está presente en un gran número de iniciativas concretas de colaboración interinstitucional e interuniversitaria, de vinculación entre universidades y su entorno socioeconómico y de proyectos conjuntos de formación." (Ferrer, 2009). La propuesta es convergente con el paradigma de la Universidad del Tercer Milenio, porque considera investigación y enseñanza indisociables epistemológicamente. Sin duda, esto proporcionará tanto a nuestros acuerdos como a nuestros equipos internacionales un gran avance. En la declaración de Cartagena, 2008, está previsto también "el establecimiento de instrumentos de comunicación para favorecer la circulación de la información y del aprendizaje", elemento fundamental en la sociedad red, concepto formulado por Castells (1999). 


\section{PROPUESTA PARA LA UNIVERSIDAD DEL TERCER MILENIO}

Bauman (2005) rescata de la antigüedad griega "la noción de Paideia" para la idea de "educación para toda la vida." Afirma que: "en el ambiente líquido moderno la educación y el aprendizaje... deben ser continuos... pues la 'formación' (destaque del autor) 'de los yos o personalidades' demanda una formación permanente y eternamente inconclusa." En este contexto, la idea de la investigación como base para la enseñanza promoverá en los atores de la educación, docentes y alumnos, la posibilidad de permanecer en formación permanente. La transformación del educador solo ocurre a través de la investigación. Por medio de la investigación constante el educador se educa y se vuelve así capaz de educar. Dejando disponible para los alumnos los nuevos conocimientos adquiridos a través de sus investigaciones, como así también los métodos e instrumentos de investigación para que estos alumnos se auto eduquen, el educador proporciona los medios necesarios para la educación para toda la vida. El poder del conocimiento y la actualización constante a través de la investigación pueden mitigar la angustia existencial y, de acuerdo con Bauman (2005), "la agonía de la elección....que siempre atormentó al Homo eligens, el hombre que escoge"...y citando Wojciechowski, este autor observa: "antiguamente un diploma universitario ofrecía un salvoconducto para la práctica de la profesión hasta la jubilación... pero esto es ahora cosa del pasado. Hoy en día, el conocimiento precisa ser constantemente renovado, las propias profesiones precisan cambiar; caso contrario, todo el esfuerzo...será inútil". En el paradigma de la universidad emergente del tercer milenio no está previsto solamente que ésta sea un lugar de transmisión del conocimiento acumulado, sino también que la propia enseñanza sea estructurada en bases más relevantes, es decir, que la producción de conocimiento se realice a partir de la investigación institucional. Es innegable que "la universidad vive una ruptura epistemológica, que configura su nuevo paradigma: que la investigación es la propia enseñanza." (Lucchesi, 2006).

La cuestión que se impone en este momento es que la expansión de la enseñanza superior no cause una pérdida de la excelencia, aquí conceptualizada como "tradición \& innovación". Esto será posible en la medida en que la universidad y las otras instituciones de enseñanza superior que complementan la oferta de cursos se mantengan fieles al ethos de la universidad - su esencia - que es la producción y difusión del conocimiento. Vivimos en una época de crisis y de cambio de paradigmas. Dentro de la gran crisis de la transformación del mundo del trabajo, de las comunicaciones y del comercio, hay una reevaluación de algunos aspectos de la posmodernidad, o "modernidad líquida" como Bauman (2001) denomina este comienzo del siglo XXI. Lucchesi y Malanga (2009) afirman que la "universidad del tercer milenio es local y global (glocal), inter y transdisciplinaria, productora del conocimiento y formadora de profesionales capaces de crear y no solo de repetir conocimiento" y que al internacionalizarse, la universidad reafirmará sus valores, funciones y misión. En este contexto de ruptura, la universidad debe contribuir con la producción de conocimiento nuevo. Y si el estado nacional producto de la modernidad está en crisis, éste es el momento de la emergencia de la organización internacional. Desde sus orígenes en la Europa Medieval, la universidad siempre fue supranacional. Tiene la misión de continuar siendo la productora y socializadora del conocimiento, razón de ser de su historia, definición de su episteme y justificativa de su ethos.

\section{CONCLUSIONES}

Un nuevo orden mundial presupone un nuevo orden educacional. En esta acción transnacional, para que la integración económica y política sea exitosa, es necesaria la integración de la educación, en especial de las universidades. Son éstas las que deben producir el conocimiento, la crítica y la innovación tecnológica, contribuyendo para la transformación social.

Ya hace mucho tiempo que la legislación brasileña reconoce una característica de la universidad: La enseñanza, la investigación y la extensión de servicios a la comunidad no están disociadas. Ahora, la integración latinoamericana está dando un paso al frente al entender que la investigación está en la base de la enseñanza superior de excelencia. Esto genera una transformación epistemológica. 
Debemos reafirmar, por lo tanto, que la internacionalización de la universidad debe considerar que su episteme y pertinencia es la producción e intercambio del conocimiento científico. $Y$ teniendo en cuenta que el conocimiento se tornó un bien permanente para el "sujeto" de la posmodernidad, la producción conjunta del conocimiento científico latinoamericano contribuirá para la superación de la injusticia y la transformación social.

\section{REFERENCIAS}

Academic Ranking of World Universities Shanghai University, China (2008). http://www.arwu.org/. Acceso: 09/02 (2011)

Bauman, Z., Modernidade e ambivalência, Jorge Zahar Editor, Rio de Janeiro, Brasil (1999).

Bauman, Z., Vida Líquida, Jorge Zahar Editor, Rio de Janeiro, Brasil (2005).

Bauman, Z.,, Entrevista. (Maria Lúcia Garcia Pallares-Burke), Tempo social: 16 (1) Brasil. http://www.scielo.br/scielo.php?pid=S0103-20702004000100015\&script=sci_arttext. Acceso: 20/07(2008).

Brasil. Ministério da Educação. Mercosul/cmc/dec. $N^{\circ}$ 17/08. Portal do MEC. http://portal.mec.gov.br/dmdocuments/dec_017_conae.pdf. Acceso: 20/04(2010).

Brasil. Ministério da Educação. Estrangeiros têm vagas em universidades, Portal do MEC. http://portal.mec.gov.br/. Acceso: 20/02(2009).

Brasil. Ministério da Educação. Assessoria de Comunicação Social. Unila avança a caminho da implantação. Portal do MEC. 17/12/2008. http://portal.mec.gov.br/. Acceso: 20/02(2009).

Brasil. Ministério da Educação. Educación Superior. La Educación Superior en el Sector Educativo del Mercosur - SEM . http://www.sic.inep.gov.br. Acceso: 22/02(2009).

Brasil. Presidência da República. Casa Civil. Subchefia para Assuntos Jurídicos. Decreto n. 6.729, de 12 de janeiro de 2009, y tabla de equivalencias. http://www.planalto.gov.br/. Acceso 20/02(2009).

Carnoy, M., Globalization, educational trends and the open society. Education Support Program. OSI Education Conference 2006: "Education and Open Society: A Critical Look at New Perspectives and Demands". Fuente: base de datos del Open Society Institute, EE.UU. (2006).

Castells, M., A Era da Informação: economia, sociedade e cultura, 3, 411-439, Paz e terra, São Paulo, Brasil (1999).

Declaración de la Conferencia Regional de Educación Superior en América Latina y el Caribe, Cartagena de Indias, Colombia, 2008. http://www.cres2008.org/. Acceso: 20/02(2009).

Declaración conjunta de los Ministros de Educación del MERCOSUR y de la Coordinadora de Centrales Sindicales del Cono Sur. Ciudad Autónoma de Buenos Aires, el 8 de junio de 2010. htpp://www.mec.gov.br.Brasil. Acceso: 09/06(2010).

Duhram, E. R. y A. Simonis, "O Ensino Superior no Estado de São Paulo" in: Indicadores de Ciência e Tecnologia. 2000 a 2006, FAPESP, São Paulo, Brasil (en prensa)

Ferrer, A. T., El Espacio Iberoamericano del Conocimiento: Retos y Propuestas, Centro de Altos Estudios Universitarios de la OEI, Madrid, España (2009). 
Kingdon, J., Agendas, Alternatives and Public Policies, 3.ed., Harper Collins, Nueva York, EE.UU. (2003).

Lucchesi, M. A. S., Higher Education Policies for The XXI Century: The Future of Emerging Countries, Problems of Education in the 21st Century, 15, 90-98, Lithuania (2009).

Lucchesi, M. A. S., Education and Social Policy in Brazil: Alternatives to Higher Education. The International Journal of Interdisciplinary Social Sciences, Common Ground Publishing Pty Ltd., Melbourne, Australia (2008). http://www.SocialSciences-Journal.com Acceso: 20/09(2010).

Lucchesi, M. A. S., Políticas Públicas para a Educação Superior no contexto Sul-Americano: convergências e transformações na passagem do século, Revista Brasileira de Política e Administração da Educação, 23, 513-528, Brasil (2007).

Lucchesi, M. A. S., La Universidad Brasileña en un Contexto de Cambios Impuesto por La Globalización (Unesco: Bibliographie sélective, Internationalisation et enseignement supérieur,2006), Revista de la Educación Superior, Anales/México, V. xxxv1, 137, 99-109 (2006).

Lucchesi, M. A. S., A universidade no limiar do III milênio: desafios e tendências, Leopoldianum, São Paulo, Brasil (2002).

Lucchesi, M. A. S. y E. B. Malanga, Post-Disciplinarity: Brazilian Universities' Ethos in the Society of Knowledge, Journal of the World Universities Forum, 2, 185-198, Common Ground Publishing Pty Ltd Melbourne, Australia (2009) www.universities-journal.com

Lucchesi, M. A. S., A Superação dos Limites Disciplinares na Universidade Brasileira e a Pesquisa Ambiental, In: IV Taller Internacional Universidad, Medio Ambiente, Energia y desarrolo sustentable - 6 Congreso Internacional de Educacion Superior (Anales) (Universidad 2008), 1, 1539-1549, UNESCO, Havana, Cuba: Universidad de Habana (2008).

Machado, M. C., Brasil: Alunos poderão estudar em séries correspondentes nos países do bloco. 22 de Enero, Brasil (2009). Portal do MEC. www.mec.gov.br. Acceso: 30/01(2009).

Morin, E., A religação dos saberes: o desafio do século XXI, Bertrand Brasil, Rio de Janeiro, Brasil (2001).

Mattelart, A., Sociedade do conhecimento e controle da informação e da comunicação, In: Encontro Latino de Economia Política da Informação, Comunicação e Cultura, 5, Salvador, Brasil. 9-11 nov. 2005. http://www.gepicc.ufba.br/enlepicc/ArmandMattelartPortugues.pdf. Acceso: 18 Enero 2008.

Performance Ranking of Scientific Papers for World Universities, del Higher Education Evaluation \& Accreditation Council of Taiwan.(2008) http://www.arwu.org/rank2008/ARWU2008_E(EN).htm. Acceso: 12/05 (2009).

Sampaio, H., Trajetória e tendências recentes do setor privado do ensino superior no Brasil. Jun.(2000), http://www.ilea.ufrgs.br/cipedes/jun00/sampaio.rtf. Acceso: 6 Abr. (2009)

Times Higher Education's 2010-2011 World University Rankings United Kingdom. http://www.timeshighereducation.co.uk/ Acceso: 09/02 (2011)

UNESCO, Comissão da Universidade de Integração Latino-Americana toma posse, Brasil 06/03 (2008). www.mec.gov.br Acceso: 08/03 (2008). 